\title{
Critical analysis of the charge-state dependence of the energy loss of channeled ions
}

\section{Citation}

Golovchenko, J. A., D. E. Cox, and A. N. Goland. 1982. Critical Analysis of the Charge-State Dependence of the Energy Loss of Channeled Ions. Physical Review B 26, no. 5: 2335-2340. doi:10.1103/physrevb.26.2335.

\section{Published Version}

doi:10.1103/PhysRevB.26.2335

\section{Permanent link}

http://nrs.harvard.edu/urn-3:HUL.InstRepos:29407051

\section{Terms of Use}

This article was downloaded from Harvard University's DASH repository, and is made available under the terms and conditions applicable to Other Posted Material, as set forth at http:// nrs.harvard.edu/urn-3:HUL.InstRepos:dash.current.terms-of-use\#LAA

\section{Share Your Story}

The Harvard community has made this article openly available.

Please share how this access benefits you. Submit a story.

Accessibility 


\title{
Physical ReVIEW B \\ CONDENSED MATTER
}

THIRD SERIES, VOLUME 26, NUMBER 5

1 SEPTEMBER 1982

Critical analysis of the charge-state dependence of the energy loss of channeled ions

\author{
J. A. Golovchenko \\ Bell Laboratories, Murray Hill, New Jersey 07974 \\ D. E. Cox and A. N. Goland \\ Brookhaven National Laboratory, Upton, New York 11973
}

(Received 26 January 1982)

\begin{abstract}
A critical analysis of channeled-ion energy-loss experiments is presented with the goal of commenting on the presence of the Barkas effect of $Z_{1}^{3}$ corrections to the stopping power. Accurate charge-density values are obtained for silicon and used to evaluate Bloch and straggling effects in the data. The remaining contributions to the data show a clear $Z_{1}^{3}$ dependence that can be explained with an electron-gas model for the Barkas effect.
\end{abstract}

Recently a comprehensive set of measurements showed that swift and relatively highly charged projectiles channeling through thin silicon crystals lose energy at a rate strictly proportional to the square of that projectile atomic number. ${ }^{1}$ This would seem somewhat strange in view of the current intense interest in the so-called higher-order $Z_{1}$ dependence on stopping power or Barkas effect. ${ }^{2-7}$. In the following we shall try to clarify and elaborate further on the status of these experiments and their implications to our understanding of stopping phenomena. In particular we wish to show that agreement between theory and experiment is not obtained unless account is taken of target polarization which is the root cause of the Barkas effect.

In Ref. 1 the stopping power $S$ (which is defined as the energy loss per unit path length divided by the target atomic density) for totally stripped ions of atomic number 9, 12, 14, 16, and 17 was measured for projectiles penetrating down the center of 〈110) axial channels in silicon at $3.086 \mathrm{Mev} / \mathrm{amu}$. The results of that work are shown in Fig. 1. Within the experimental uncertainties the stopping power is seen to scale as the square of the atomic number $Z_{1}$. The stopping power for the best channeled ions was determined by extrapolating the sharp least-energy-loss side of the distribution to the baseline linearly. Effects of energy-loss straggling were argued to be small based on the anticipated very-low-electron density along the best channeled track. It was further noted that in contrast with most other experiments of this type, here the ratio of $Z_{1}$ to $v$, the velocity of the incident ion, was so large that a significant contribution to the stopping

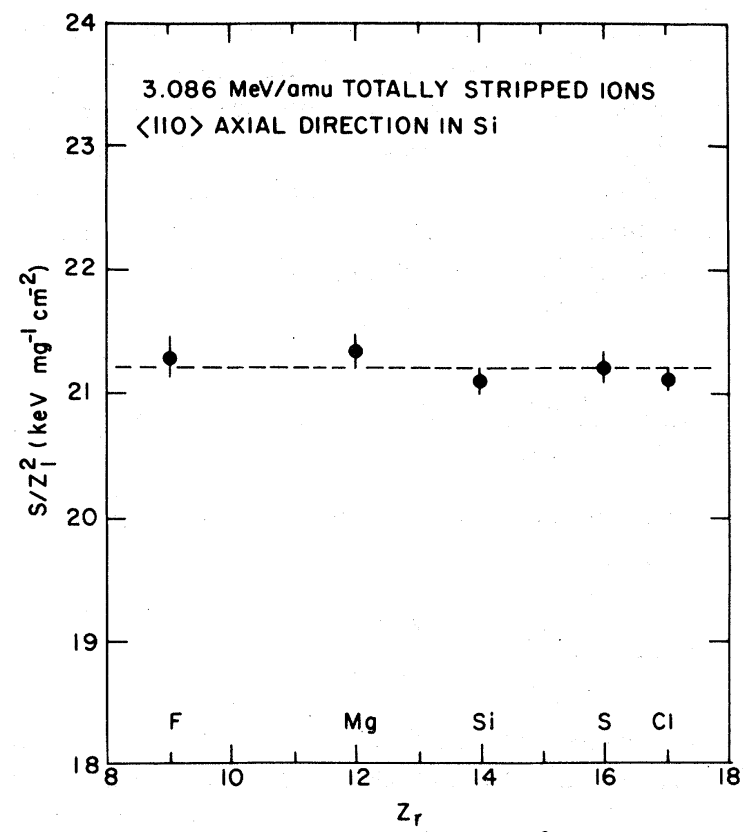

FIG. 1. Stopping power divided by $Z_{1}^{2}$ vs $Z_{1}$ for totally stripped ions of fluorine, magnesium, silicon, sulfur, and chlorine, channeling down a $\langle 110\rangle$ axial direction in silicon. 
from Bloch's correction could be expected. This correction turns out to also depend on the local charge density along the particle track. Furthermore, it also contributes to the expected $Z_{1}^{3}$ effect in such a way as to tend to cancel any contribution from polarization effects. Reference 1 concluded that the apparent absence of $Z_{1}^{3}$ effects in the energy-loss data was probably an "accident" with Bloch and polarization terms canceling. Based on this discussion one concluded that in order to utilize the data in Fig. 1 to comment on the existence and magnitude of polarization contributions to the stopping, a firm knowledge of both the center-channel average charge density and the stopping effects depending on it must be achieved. Thus in the following we give a more comprehensive discussion of these "local" effects and the results of our most ambitious study of the midchannel charge density in silicon. This analysis shows that a significant polarization effect must be present and that a small modification of the electron-gas model discussed by Lindhard ${ }^{6}$ and Esbensen ${ }^{8}$ can account for it.

The charge density averaged along the $\langle 110\rangle$ axial direction in silicon is shown in Fig. 2. The results have been generated from a bond charge model derived from a recent refinement of the electrondensity distribution using $\mathrm{x}$-ray data obtained by Pendellösung-fringe measurements. They represent probably the best known charge-density values for any crystal. The details of how the Fourier recomposition of this density was effected are described in the Appendix. The main value we shall require from Fig. 2 is the midchannel density which is $\rho_{\text {loc }}=0.045 e \AA^{-3}$. This is significantly below the homogeneous valence gas value of 0.2 used in many previous analyses of channeled stopping in sil-

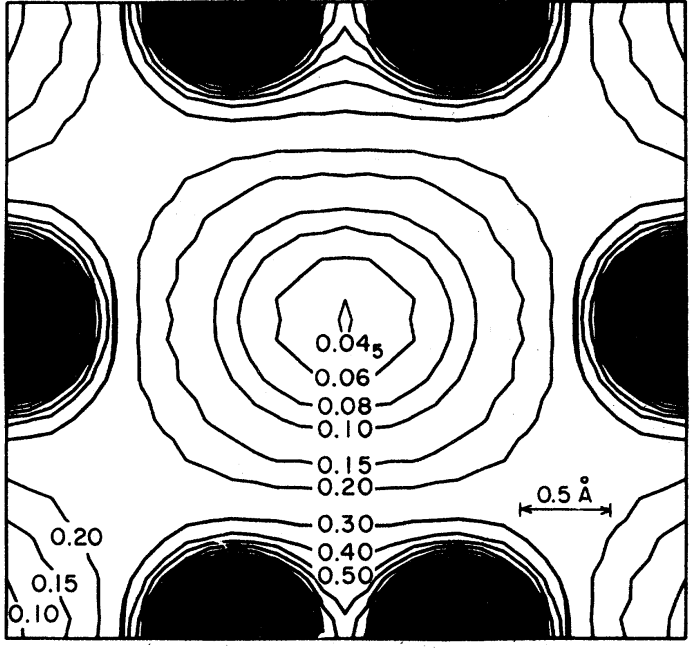

FIG. 2. Contour plot of average electron charge density in $e$ per $\AA^{-3}$ looking down the [110] axial direction in silicon.

icon. ${ }^{9,10}$ It is also smaller than a Thomas-Fermi value of 0.067 that was used in Ref. 1. Dr. M. Schlüter of Bell Laboratories has obtained a value of $0.042 e \AA^{-3}$ by a self-consistent pseudopotential calculation.

Armed with what we now believe to be a very accurate knowledge of the charge density in silicon we proceed with a brief but illuminating derivation of the classic Beth-Bloch energy-loss formula which will serve as the foundation of our current discussion. ${ }^{11}$ Imagine a free electron of momentum $\hbar k=m v$ incident on a totally stripped ion of charge $Z_{1}$ e. A knowledge of the scattering angle distribution in this frame is sufficient to evaluate the energy-loss rate of the heavy particle in the lab frame through the formula

$$
\frac{d E}{d x}=\frac{N Z_{2} \hbar^{2}}{m} 2 \pi \int_{-1}^{1} d(\cos \theta) \sin ^{2} \frac{\theta}{2}\left|\sum_{l=0}^{\infty}(2 l+1)\left(e^{2 i \delta_{l}}-1\right) P_{l}(\cos \theta)\right|^{2} .
$$

Here $N Z_{2}$ is the density of electrons in the target, $\theta$ is the electron scattering angle in the stationary projectile frame, and

$$
\delta_{l}=\arg \Gamma\left[l+1-\frac{Z_{1} e^{2}}{\hbar v} i\right),
$$

are the well-known Coulomb phase shifts for angular-momentum quantum number $l$. Curiously enough, Eq. (1) may be evaluated exactly yielding the following angular-momentum expansion for the energy-loss rate:

$$
\frac{d E}{d x}=\frac{4 \pi N Z_{2} e^{4} Z_{1}^{2}}{m v^{2}} \sum_{l=0}^{\infty} \frac{(l+1)}{(l+1)^{2}+\kappa^{2} / 4} .
$$

Here $\kappa$ is $Z_{1} e^{2} / \hbar v$, known as Bohr's parameter. This result is exact and not widely known. It is also loga- 
rithmically divergent as the sum is extended to infinity. This is of course related to the infinite range of the Coulomb potential. Before adding the necessary physics to remedy this situation we break up the summation in Eq. (3) to make it read

$$
\frac{d E}{d x}=\frac{4 \pi N Z_{2} e^{4} Z_{1}^{2}}{m v^{2}}\left(\sum_{l=0}^{l_{\max }} \frac{1}{(l+1)}-\frac{\kappa^{2}}{4} \sum_{l=0}^{\infty} \frac{1}{(l+1)\left[(l+1)^{2}+\left(\kappa^{2} / 4\right)\right]}\right) .
$$

From the definition of the digamma function $\Psi,{ }^{12}$ Eq. (4) may be rewritten

$$
\begin{aligned}
\frac{d E}{d x} & =\frac{4 \pi N Z_{2} e^{4} Z_{1}^{2}}{m v^{2}}\left[\sum_{l=0}^{l_{\max }} \frac{1}{(l+1)}+\Psi(1)-\operatorname{Re} \Psi\left[1+\frac{i \kappa}{2}\right)\right] \\
& =\frac{4 \pi N Z_{2} e^{4} Z_{1}^{2}}{m v^{2}}\left[\Psi\left(l_{\max }+2\right)-\operatorname{Re} \Psi\left(1+\frac{i \kappa}{2}\right)\right] .
\end{aligned}
$$

This formula reminds us of the standard nonrelativistic Bethe-Bloch result ${ }^{13,14}$ if $^{12}$

$$
\Psi\left(l_{\max }+2\right) \rightarrow \ln \frac{2 m v^{2}}{\hbar \omega} .
$$

Now note that this association is all the more reasonable when the angular-mometum sum in Eq. (5) is limited by the adiabaticity condition that collisions leading to excitation can be of duration no longer than a characteristic orbit time $2 \pi / \omega$ ( $\omega$ being effective oscillator frequency); i.e.,

$$
\Delta t=\frac{\hbar l_{\max }}{2 m v^{2}} \sim \frac{1}{\omega} \rightarrow l_{\max } \sim \frac{2 m v^{2}}{\hbar \omega} .
$$

The Bethe-Bloch result is then completely recovered if the proper oscillator strength is included for each effective oscillator in the target atom.

In the deduction of these results perturbation theory has not been used. Also, the angularmomentum sums give an idea as to which electrons are contributing to the stopping of channeled particles. Consider for example the Bloch contribution, i.e., the second term in Eq. (4). For the experiments under discussion $\kappa^{2} / 4$ was always less than one. Contributions to the sum come from the smallest values of $l$ only. (This is also why convergence difficulties did not have to be dealt with for this term.) For $l=0-1$ this corresponds to impact parameters $\sim \hbar / m v \sim 0.05 \AA$ between target electrons and the projectile. This is the basis for the claim that Bloch corrections depend upon the electron density directly on the path of the channeled particle. Thus for this term in the stopping we obtain

$$
-\frac{\rho_{\mathrm{loc}}}{N Z_{2}} \frac{\kappa^{2}}{4} \sum_{l=0}^{\infty} \frac{1}{(l+1)\left[(l+1)^{2}+\kappa^{2} / 4\right]} .
$$

A more exact expression might take the form

$$
-\frac{1}{N Z_{2}} \frac{\kappa^{2}}{4} \sum_{l=0}^{\infty} \rho(l) \frac{1}{(l+1)\left[(l+1)^{2}+\kappa^{2} / 4\right]}
$$

with $\rho(l)$ being the average charge density at a distance $h(l+1) / m v$ from the track. Also no contributions from electrons past the adiabatic limit (i.e., core electrons) should be included.

The contribution of the Bethe term may be estimated for the channeling conditions of the experiments by noting that the adiabatic distances connected with the natural frequencies of the silicon $L$ electrons are already smaller than the distance from any atom to the center of the $\langle 110\rangle$ channel (which is where the particle track lies). Hence we must mainly deal with the valence-electron oscillator strength distribution to evaluate the stopping. As an initial estimate we can imagine that the valence gas is homogeneous and all the dipole oscillator strength collects at the plasma frequency

$$
\omega_{p}=\left(\frac{4 \pi \rho_{\mathrm{hom}} e^{2}}{m}\right)^{1 / 2} \text {. }
$$

The Bethe contribution for the homogeneous valence gas then gives a value of $39.5 Z_{1}^{2} \mathrm{keV} \mathrm{mg}^{-1}$ $\mathrm{cm}^{-2}$; almost twice the experimental value. The major reason for this discrepancy is not hard to find. Indeed, we expect a strong reduction in the close-collision contribution in this term because the gas is not uniform. The long-wavelength plasmon contribution alone can be evaluated separately through the formula ${ }^{15}$

$$
\frac{d E}{d x}=\frac{Z_{1}^{2} e^{2} \omega_{p}^{2}}{v^{2}} \ln \frac{v}{v_{f}}
$$

$$
\left[v_{f}=(\hbar / m)\left(\pi^{2} \rho_{\mathrm{hom}}\right)^{1 / 3}\right] \text {, }
$$


and we find that $40 \%$ of the homogeneous gas loss goes into these long-wavelength excitations. If we assume the close-collision contribution for our inhomogeneous gas is reduced by the ratio of the actual charge density to the uniform gas value we deduce a stopping value of $24 Z_{1}^{2} \mathrm{keV} \mathrm{mg}^{-1} \mathrm{~cm}^{-2}$ : a considerable improvement. With this encouraging result we interpret all the $Z_{1}^{2}$ contribution as coming from the Bethe term and continue our analysis.

There is another straighforward correction that we can apply now that we have a reliable charge density for the channel. That is the straggling error in the leading edge least-energy-loss analysis of the data. The variance in energy loss is also a local effect and is given by ${ }^{16}$

$$
\Omega=\left(4 \pi Z_{1}^{2} e^{4} \rho_{\mathrm{loc}} T\right)^{1 / 2},
$$

where $T$ is the target thickness. The widths predicted by this equation are generally smaller than the extent of the linear regions from which the least energy loss was obtained. Hence we confirm the negligible contribution of straggling.

Figure 3 shows a plot together with the Bloch contribution. The value of the Bethe term has been adjusted so the theoretical curve passes through the last data point. It is clear that some positive sloping correction is necessary to bring the $Z_{1}^{3}$ dependence of the energy loss into agreement with the experiment.

Such a contribution can result from the fact that the actual orbits of electrons in the Coulomb field of the projectile are not hyperbolic. There are two possible causes for this. One is the effect of the binding of the electrons to the target atoms. ${ }^{2-5}$ The

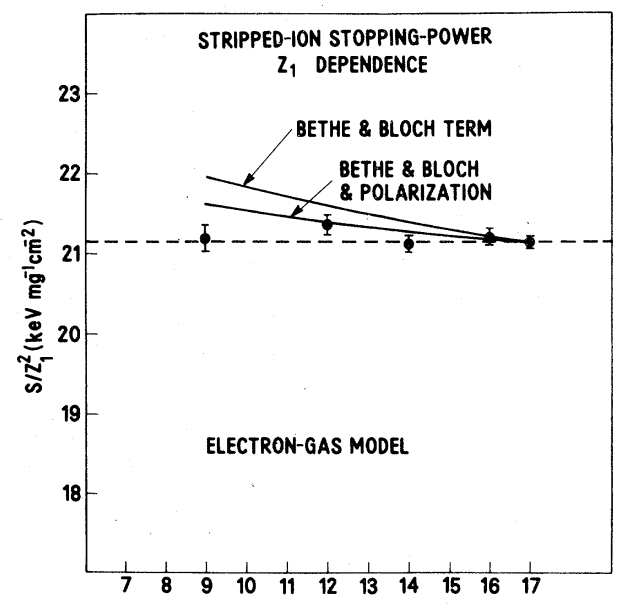

FIG. 3. Stopping power divided by $Z_{1}^{2}$ vs $Z_{1}$. Theoretical curves are presented with and without polarization term in Eq. (14). The values of $\alpha$ have been chosen so curves pass through the last data point. second lies in electron correlation effects. ${ }^{6,8}$ That is, an electron scattering sees not only the field of the projectile but that of other electrons also scattering from the projectile. Lindhard ${ }^{6}$ has argued heuristically that simple screening can result in $Z_{1}^{3}$ effects through a change in the self-energy of the projectile during passage through the target. This self-energy effect is due to the polarization cloud the projectile drags along with it. After carrying out the appropriate renormalizations of relative velocities and angular momentum, Lindhard finds

$$
\left.\frac{d E}{d x}\right|_{\mathrm{pol}}=\frac{Z_{1}^{2} e^{2} \omega_{p}^{2}}{v^{2}}\left[\frac{3 \pi}{2} \frac{Z_{1} e^{2} \omega_{p}}{m v^{3}} \ln \frac{m v^{2}}{\hbar \omega_{p}}\right],
$$

where $\omega_{p}$ is again the plasma frequency of the gas. As opposed to virtually all published results based on the target-atom binding effect, it is emphasized that nearly equal contributions to this result come from close and distant collisions. We may therefore assume that this result will be modified by the channeling effect in a way just analogous to the way the Bethe term is modified. (It is essential that only one effective nonlocalized oscillator contribute to the stopping for this to be so.)

With all of the above discussion in mind, we adopt as our final energy-loss formula:

$$
\begin{aligned}
\frac{d E}{d x}= & \alpha \frac{Z_{1}^{2} e^{2} \omega_{p}^{2}}{v^{2}}\left[\ln \frac{2 m v^{2}}{\hbar \omega_{p}}+\frac{3 \pi}{2} \frac{Z_{1} e^{2} \omega_{p}}{m v^{3}} \ln \frac{m v^{2}}{\hbar \omega_{p}}\right] \\
& -\frac{\rho_{\text {loc }}}{\rho_{\text {hom }}} \frac{Z_{1}^{2} e^{2} \omega_{p}^{2}}{v^{2}} \frac{\kappa^{2}}{4} \\
& \times \sum_{l=0}^{\infty} \frac{1}{(l+1)\left[(l+1)^{2}+\kappa^{2} / 4\right]} .
\end{aligned}
$$

Figure 3 shows a comparison of the experimental data from Ref. 1 with the predictions of the last equation. A value of alpha has been chosen to make the curve go through the chlorine data point (which has the smallest error bar). The values of alpha for all the plots are $\sim 0.54$ and $\hbar \omega_{p}$ is taken as $16.6 \mathrm{eV}$ and $\rho_{\mathrm{loc}} / \rho_{\text {hom }}=0.045 / 0.2$. With the recent work on charge density reported here and the discussion of theory given earlier, we believe the Bloch term to be the most reliable part of the last expression. Except for the value of alpha, the $Z_{1}^{2}$ dependence of the stopping must certainly be contained in the Bethe term. Inspection of Fig. 3 shows that the electron-gas prediction of the polarization effect using just the valence gas improves the overall agreement with the data substantially. Indeed, a slightly 
larger polarization correction could be tolerated.

We conclude that decomposition of data for the energy loss of channeled ions versus $Z_{1}$ into its various components, i.e., Bethe, Bloch, and polarization (Barkas), can be effected if accurate local charge densities on the ion track are independently known. When this decomposition, procedure is applied, a polarization contribution within a factor of 2 of theoretical electron-gas estimates is required to account for the data. We take this result to be evidence of the first observation of the Barkas effect in a valence gas. We refrain from commenting further on more quantitative questions such as theoretical prediction of the observed value of alpha since this approach represents a crude attempt to extend the homogeneous gas model to clearly inhomogeneous problem. Spurred on by the realization that channeling measurements can be sensitive to higherorder $Z_{1}$ effects we hope that further theoretical work along these lines will be attempted. Finally we note that the cancellation we believe explains the apparent absence of higher-order $Z_{1}$ effects in the stopping should not be so complete for other channeling directions. Indeed, should higher-order effects still be unobservable in cases where the minimum charge density differs significantly from $0.045 e \mathrm{~cm}^{-3}$ a rethinking of the polarization question would be appropriate.
The work performed at BNL was supported by the U. S. Department of Energy under Contract NO. DE-AC02-76CH00016.

\section{APPENDIX: CALCULATION OF ELECTRON DENSITIES}

Electron-density diagrams were obtained by Fourier transformation of the structure factors of 293 reflections, corresponding to $\sin \theta / \lambda<2.0$, calculated on the basis of the bond-charge model proposed by Scheringer. ${ }^{17}$ This involves a spherically symmetric core with a net charge $q(\mathrm{Si})$ of 1.12 positive charge units and negative Gaussian-distributed charge clouds of $0.56 e$ at the bond center between near-neighbor $\mathrm{Si}$ atoms to describe the covalent bonds. Variable parameters are introduced to allow for anisotropic vibration of the bond charges and contraction of the valence shell. The scattering factor for silicon $f(\mathrm{Si})$ is set up as the sum of the scattering factor of the core $f\left(\mathrm{Si}^{4+}\right)$ and that the valence shell, which involves the difference between the neutral atom scattering factor $f\left(\mathrm{Si}^{0}\right)$ and $f\left(\mathrm{Si}^{4+}\right)$ modified to allow for the contraction due to the net positive charge on the atom. Values of $f\left(\mathrm{Si}^{0}\right)$ and $f\left(\mathrm{Si}^{4+}\right)$ were obtained by means of the analytic approximation given in Ref. 18. For the sake of comparison, a second set of structure factors was calcu-

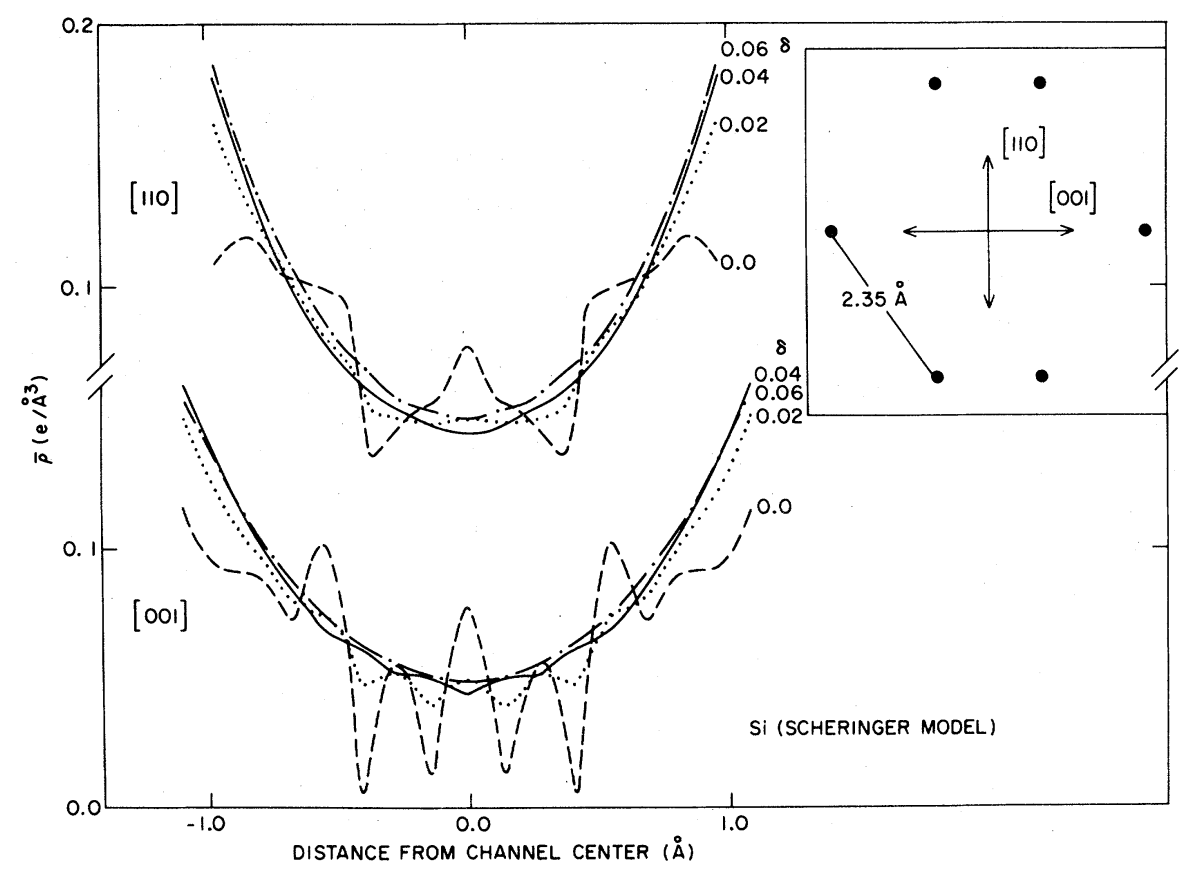

FIG. 4. Charge densities in a (110) plane of silicon as a function of distance from the center of the channel along the [110] and [001] directions. Plots are shown for various values of $\delta$. 
lated from the valence scattering factors listed in Ref. $18, f\left(\mathrm{Si}^{\mathrm{val}}\right)$, which are obtained after repopulation of the orbitals to an $s p^{3}$ configuration.

In order to investigate the effect of seriestermination errors, the procedure described by
Weiss ${ }^{19}$ was followed. In this, the average charge density centered about a small parallelepiped with edges $2 \delta a, 2 \delta b$, and $2 \delta c$ is calculated instead of the point density. The averaged density is given by the expression

$$
\bar{\rho}(X Y X)=\frac{1}{V} \sum_{h} \sum_{k}^{\infty} \sum_{1} F(h k l) \exp \left[-2 \pi_{i}(h X+k Y+1 Z)\right] \frac{\sin 2 \pi h \delta}{2 \pi h \delta} \frac{\sin 2 \pi k \delta}{2 \pi k \delta} \frac{\sin 2 \pi l \delta}{2 \pi l \delta}
$$

Some value of $\delta$ is then sought such that the series oscillations are suitably damped without the loss of significant detail. Some illustrative examples of this process applied to the analysis of magnetic spin densities are given by Moon. ${ }^{20}$

Some of the results of this averaging procedure applied to Fourier projections on a (110) plane of $\mathrm{Si}$ are shown in Fig. 4. The two sets of curves show $\bar{\rho}$ as a function of distance from the channel center in the [001] and [1̄o] directions in that plane (as indicated in the inset to the figure) for various values of $\delta$. The series oscillations are quite pronounced for $\delta=0.0$, but are essentially damped out for $\delta=0.04$ and above.

Figure 2 shows the average charge density seen by a channeled particle in the [110] direction, i.e., Eq. (15) averaged along the [110] direction. A value of $\delta=0.04$ is used. It may therefore be concluded that the average electron density derived from the Scheringer model is about $0.045 \pm 0.005 e \AA^{-3}$ at the center of the channels. At a distance of $0.4 \AA$ from the center, $\bar{\rho}$ is about $0.06 e \AA^{-3}$. For comparison, the value at the channel centers for the simple valence model is significantly higher, $0.08+0.01 e$ $\AA^{-3}$.
1J. A. Golovchenko, A. N. Goland, J. S. Rosner, C. E. Thorn, H. E. Wegner, H. Knudsen, and C. D. Moak, Phys. Rev. B 23, 957 (1981).

2J. C. Ashley, R. H. Ritchie, and W. Brandt, Phys. Rev. B ㄴ, 2393 (1972).

3J. D. Jackson and R. L. McCarthy, Phys. Rev. B $\underline{6}, 4131$ (1972).

${ }^{4}$ J. C. Ashley, R. H. Ritchie, and W. Brandt, Phys. Rev. A $\underline{8}, 2402$ (1973).

${ }^{5}$ K. W. Hill and E. Merzbacher, Phys. Rev. A 9, 156 (1974).

6J. Lindhard, Nucl. Instrum. Methods 132, 1 (1976).

${ }^{7}$ W. H. Barkas, N. J. Dyer, and H. H. Heckman, Phys. Rev. Lett. 11, 26 (1963).

${ }^{8}$ H. Esbensen, Ph.D. thesis, Aarhus University, Aarhus, Denmark, 1977 (unpublished).

${ }^{9}$ B. R. Appleton, C. Erginsoy, and W. M. Gibson, Phys. Rev. 161, 330 (1967).

${ }^{10}$ F. Bonsipnori and A. Desalvo, J. Phys. Chem. Soc. 31,
2191. (1970).

${ }^{11}$ The possibility of this derivation was indicated to one of us (J.A.G.) some years ago by Professor J. Lindhard.

${ }^{12}$ Handbook of Mathematical Functions, edited by $\mathbf{M}$. Abramowitz and I. A. Stegun (Dover, New York, 1966).

${ }^{13}$ H. A. Bethe, Ann. Phys. (Leipzig) $\underline{\mathbf{5}}, 325$ (1930).

${ }^{14}$ F. Bloch, Ann. Phys. (Leipzig) 16, 285 (1933).

${ }^{15}$ D. Pines Elementary Excitations in Solids (Benjamin, New York 1963).

16J. Lindhard, K. Dan. Vidensk. Selsk. Mat.-Fys. Medd. 34 (14) (1965).

${ }^{17}$ C. Scheringer, Acta Crystallogr. A $\underline{36}, 210$ (1980).

${ }^{18}$ International Tables for X-ray Crystallography, edited by N. F. M. Henry and K. Lonsdale (Kynoch, Birmingham, England, 1965), Vol. 1.

${ }^{19}$ R. J. Weiss, X-ray Determination of Electron Distribution (North-Holland Amsterdam, 1966), p. 72.

${ }^{20}$ R. M. Moon, Int. J. Magn. 1, 219 (1971). 